\title{
ON THE LEGENDRIAN AND TRANSVERSE CLASSIFICATION OF CABLINGS
}

\author{
BÜLENT TOSUN
}

\begin{abstract}
In this paper, we study Legendrian and transverse knots in the knot type of a $(p, q)$-cable of a knot $\mathcal{K}$ in $S^{3}$. We give two structural theorems that describe when the $(p, q)$ - cable of a Legendrian simple knot type $\mathcal{K}$ is also Legendrian simple.
\end{abstract}

\section{Introduction}

Legendrian and transverse knots are not just natural objects of study in contact 3-manifolds but also important in capturing the geometry and topology of underlying contact structure. For example, the tight versus overtwisted dichotomy is a result of having a bound on the classical invariants $\operatorname{tb}(L)$ and $\mathrm{r}(L)$ associated with a Legendrian knot $L$ in tight contact structures, see $[1,2,6]$. A similar statement is true for transverse knots in a given knot type and for their unique classical invariant, self-linking number $s l$. Hence, one wants to better understand the classification of Legendrian and transverse knots. In particular, one naturally wonders if, given topological knot type, tb and $\mathrm{r}$ (respectively $s l$ ) determine the Legendrian (respectively transverse) isotopy class of Legendrian knots representing this knot type. Such a knot type is called Legendrian (respectively transverse) simple and non-simple otherwise. This problem has been worked out on some nice class of knots $[7,10,13]$ and under certain topological operation on certain knot types $[9,11]$. In this paper, we continue the study of cabling begun in [9].

1.1. Cabling. Recall the $(p, q)$-cable of a knot type $\mathcal{K}$, denoted $\mathcal{K}_{(p, q)}$, is the knot type obtained by taking the isotopy class of a $(p, q)$-curve on the boundary of a tubular neighborhood of a representative of $\mathcal{K}$ (where $p$ denotes the meridional winding and $q$ denotes longitudinal winding). We will also denote this $(p, q)$-curve by the fraction $\frac{q}{p}$.

In [9], Etnyre and Honda studied the Legendrian and transverse classification of cables of a knot in $\left(S^{3}, \xi_{s t d}\right)$ that satisfy a property called the uniform thickness property (UTP). They proved that $\mathcal{K}_{(p, q)}$ is Legendrian simple if $\mathcal{K}$ is Legendrian simple and uniformly thick. The UTP is, for example, satisfied by negative torus knots [9] and known to be preserved under the cabling operation [9], [20]. On the other hand, the unknot and positive torus knots $[9,12]$ are some examples of nonuniformly thick knot types. Indeed, by using the fact that the $(2,3)$-torus knot is not uniformly thick, Etnyre and Honda exhibit one of the first examples of a transversely non-simple knot type, the $(2,3)$-cable of the $(2,3)$-torus knot (cf. [3]). Finally, in [12],

Received by the editors August 31, 2012. 
Legendrian and transverse cables of positive torus knots were completely classified, using, in part, the results in this paper.

Aim: In this paper, we study Legendrian and transverse simplicity for cables of a knot type $\mathcal{K}$ which is not necessarily uniformly thick. This assumption led us to study two knot invariants the contact width and the lower width, the first of which was already introduced and studied in [9].

1.2. The contact width and sufficiently positive cables. Given a tight contact manifold $(M, \xi)$, let $\mathcal{K}$ be a topological knot type and $\mathcal{L}(\mathcal{K})$ be the set of Legendrian isotopy classes of $\mathcal{K}$. As the Thurston-Bennequin invariant of a knot $L$ in $\mathcal{L}(\mathcal{K})$ is bounded above $[2,6]$, we may then define the maximal Thurston-Bennequin number of a knot type $\mathcal{K}$ to be

$$
\overline{\operatorname{tb}}(\mathcal{K})=\max \{\operatorname{tb}(L) \mid L \in \mathcal{L}(\mathcal{K})\} .
$$

The contact width of a knot type is given by

$$
\omega(\mathcal{K})=\sup \frac{1}{\operatorname{slope}\left(\Gamma_{\partial\left(S^{1} \times D^{2}\right)}\right)},
$$

where the supremum is taken over all $S^{1} \times D^{2} \hookrightarrow S^{3}$ representing $\mathcal{K}$ with $\partial\left(S^{1} \times D^{2}\right)$ convex. In order to make sense of slopes of homotopically non-trivial curves on $\partial\left(S^{1} \times D^{2}\right)$ we identify $\partial\left(S^{1} \times D^{2}\right)=\mathbb{R}^{2} / \mathbb{Z}^{2}$ where the meridian has slope 0 and the well-defined longitude (as $\mathcal{K}$ is in $S^{3}$ ) has slope $\infty$.

Our first main result can now be stated as follows.

Theorem 1.1. If $\mathcal{K}$ is Legendrian simple and $\omega(\mathcal{K}) \in \mathbb{Z}$. Then its $(p, q)$-cable, $\mathcal{K}_{(p, q)}$, is also Legendrian simple and admits a classification in terms of the classification of $\mathcal{K}$, provided $\frac{p}{q}>\omega(\mathcal{K})$. Moreover, the maximal Thurston-Bennequin invariant is

$$
\overline{\operatorname{tb}}\left(\mathcal{K}_{(p, q)}\right)=p q-\left|\overline{\operatorname{tb}}(\mathcal{K}) \bullet \frac{p}{q}\right|,
$$

and the set of rotation numbers realized by $L_{(p, q)} \in \mathcal{L}\left(\mathcal{K}_{(p, q)}\right)$ with $\operatorname{tb}(L)=$ $\overline{\operatorname{tb}}\left(\mathcal{K}_{(p, q)}\right)$ is

$$
\{q \cdot \mathrm{r}(K) \mid K \in \mathcal{L}(\mathcal{K}), \operatorname{tb}(K)=\overline{\operatorname{tb}}(\mathcal{K})\}
$$

If $K \in \mathcal{L}(\mathcal{K})$ is a non-destabilizable with $\operatorname{tb}(K)=n<\overline{\operatorname{tb}}(K)$, then there is nondestabilizable $L_{(p, q)}^{\prime}$ in $\mathcal{L}\left(\mathcal{K}_{(p, q)}\right)$ with $\operatorname{tb}\left(L_{(p, q)}^{\prime}\right)=p q-\left|\frac{1}{n} \bullet \frac{p}{q}\right|$ and the set of rotation numbers realized by such representatives is

$$
\{q \cdot \mathrm{r}(K) \mid K \in \mathcal{L}(\mathcal{K}), \operatorname{tb}(K)=n\} .
$$

1.3. The UTP, the lower width and sufficiently negative cables. A knot type $\mathcal{K}$ is said to satisfy the uniform thickness property if the following hold:

(1) $\overline{\operatorname{tb}}(\mathcal{K})=\omega(\mathcal{K})$

(2) Every embedded solid tori $S^{1} \times D^{2} \hookrightarrow S^{3}$ representing $\mathcal{K}$ can be thickened to a standard neighborhood of a maximal tb Legendrian knot.

The motivation behind this definition is the following result of Etnyre and Honda. 
Theorem 1.2 (Etnyre and Honda 2005, [9]). If $\mathcal{K}$ is knot type which is Legendrian simple and satisfies the UTP, then $\mathcal{K}_{(p, q)}$ is Legendrian simple for any $p, q$.

We say that a solid torus $S^{1} \times D^{2}$ with convex boundary representing $\mathcal{K}$ is non-thickenable, if there is no $N^{\prime}$ containing $S^{1} \times D^{2}$ (whenever we discuss solid torus contained in another we assume they have the same core) with slope $\left(\Gamma_{N^{\prime}}\right) \neq$ $\operatorname{slope}\left(\Gamma_{N}\right)$. Since there are knots with this property, see $[9,12]$, we define yet another invariant of a knot, the lower width, to be

$$
\ell \omega(\mathcal{K})=\inf \frac{1}{\operatorname{slope}\left(\Gamma_{\partial\left(S^{1} \times D^{2}\right)}\right)},
$$

where $S^{1} \times D^{2}$ ranges over all non-thickenable solid tori representing $\mathcal{K}$ with convex boundary.

Our second main result addresses classification of cables with cabling slope less than the lower width.

Theorem 1.3. If $\mathcal{K}$ is Legendrian simple and $\ell \omega(\mathcal{K}) \in \mathbb{Z}$. Then $\mathcal{K}_{(p, q)}$ is also Legendrian simple, provided $\frac{p}{q}<\ell \omega(\mathcal{K})$. Moreover,

$$
\overline{\operatorname{tb}}\left(\mathcal{K}_{(p, q)}\right)=p q=\omega\left(\mathcal{K}_{(p, q)}\right),
$$

and the set of rotation numbers realized by $L_{(p, q)} \in \mathcal{L}\left(\mathcal{K}_{(p, q)}\right)$ with $\operatorname{tb}(L)=$ $\overline{\operatorname{tb}}\left(\mathcal{K}_{(p, q)}\right)$ is

$$
\{ \pm(p+q(n+r(L)): L \in \mathcal{L}(\mathcal{K}), \operatorname{tb}(L)=-n\}
$$

where $n$ is the integer that satisfies

$$
-n-1<\frac{p}{q}<-n
$$

It is not difficult to show that for any knot type $\mathcal{K}$ the inequality $\overline{\operatorname{tb}}(\mathcal{K}) \leq \omega(\mathcal{K}) \leq$ $\overline{\operatorname{tb}}(\mathcal{K})+1$ holds. Now, if $\mathcal{K}$ is the unknot, the values for the contact width and the lower width are surprising. First of all $\omega(\mathcal{K})=\overline{\operatorname{tb}}(\mathcal{K})+1=0$ as $\overline{\operatorname{tb}}(\mathcal{K})=-1$ and the complement of the unknot in $S^{3}$ is a neighborhood of another unknot. Second, $\ell \omega(\mathcal{K})=-\infty$ as the set of non-thickenable neighborhoods of the unknot with convex boundary is empty. Hence, in the case that $\mathcal{K}$ is the unknot in Theorem 1.1 and the proof of Theorem 1.3 (see Section 4) we get the following corollary which was originally proved by Etnyre and Honda in [10].

Corollary 1.4. Torus knots are Legendrian, and hence transversely simple.

Moreover, if $\omega(\mathcal{K})=\overline{\operatorname{tb}}(\mathcal{K})=\ell \omega(\mathcal{K})$, then $\mathcal{K}$ is uniformly thick and our Theorems 1.1 and 1.3 recover Theorem 1.2 of Etnyre and Honda above.

1.4. Idea of the proof and outline. The main idea is to show under the assumptions of each of the theorem above, one understands solid tori in a given knot type well enough to classify maximal tb representatives of the cabled knot type and can always find bypass disks and hence destabilize a Legendrian knot with non-maximal tb. The necessary assumptions in the theorems was mainly motivated from the work of Etnyre and Honda in [9]. We begin, in Section 2, by collecting the necessary 


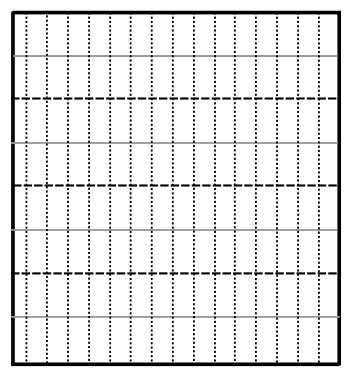

FiguRE 1. Standard convex tori. Legendrian divides are dashed. Legendrian rulings are dotted vertical, i.e., non-singular lines of slope $\infty$.

definitions, tools and facts about convex surface theory $[5,15,17]$ and about the classification of Legendrian and transverse knots [10]. With these definitions in place, we conclude, in Section 3, with the proof of Theorems 1.1 and 1.3.

\section{Preliminaries}

In this section, we will give basic definitions and the necessary background material which will be used in the rest of the paper.

2.1. Convex surfaces, bypasses and the Farey tessellation. Recall a surface $\Sigma$ in a contact manifold $(M, \xi)$ is convex if it has a neighborhood $\Sigma \times I$, where $I=(-\epsilon, \epsilon)$ is some interval, and $\xi$ is $I$-invariant in this neighborhood. Any closed surface can be $C^{\infty}$-perturbed to be convex. Moreover, if $L$ is a Legendrian knot on $\Sigma$ for which the contact framing is non-positive with respect to the framing given by $\Sigma$, then $\Sigma$ may be perturbed in a $C^{0}$ fashion near $L$, but fixing $L$, and then again in a $C^{\infty}$ fashion away from $L$ so that $\Sigma$ is convex.

Given a convex surface $\Sigma$ with $I$-invariant neighborhood, let $\Gamma_{\Sigma} \subset \Sigma$ be the multicurve where $\xi$ is tangent to the $I$ factor. This is called the dividing set of $\Sigma$. If $\Sigma$ is oriented, it is easy to see that $\Sigma \backslash \Gamma=\Sigma_{+} \cup \Sigma_{-}$where $\xi$ is positively transverse to the $I$ factor along $\Sigma_{+}$and negatively transverse along $\Sigma_{-}$. If $L$ is a Legendrian curve on a $\Sigma$ then the framing of $L$ given by the contact planes, relative to the framing coming from $\Sigma$, is given by $-\frac{1}{2}(L \cdot \Gamma)$. Moreover, if $L=\partial \Sigma$ then the rotation number of $L$ is given by $r(L)=\chi\left(\Sigma_{+}\right)-\chi\left(\Sigma_{-}\right)$.

2.1.1. Convex tori. A convex torus $T$ is said to be in standard form if $T$ can be identified with $\mathbb{R}^{2} / \mathbb{Z}^{2}$ so that $\Gamma_{T}$ consists of $2 n$ horizontal curves (note $\Gamma_{T}$ will always have an even number of curves and we can choose a parametrization to make them horizontal) and the characteristic foliations consists of $2 n$ horizontal lines of singularities ( $n$ lines of sources and $n$ lines of sinks) and the rest of the foliation is by non-singular lines of slope $s$. See Figure 1.

The lines of singularities are called Legendrian divides and the other curves are called ruling curves. We note that the Giroux Flexibility Theorem [15] allows us to $C^{0}$ isotope any convex torus into standard form. 


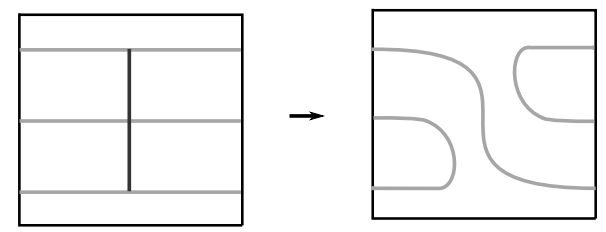

FIGURE 2. A piece of original surface $\Sigma$ with bypass arc $\alpha$, on the left. The surface $\Sigma_{1}$ after isotoping $\Sigma$ across $D$, on the right.

2.1.2. Bypasses and tori. Let $\Sigma$ be a convex surface and $\alpha$ a Legendrian arc in $\Sigma$ that intersects the dividing curves $\Gamma_{\Sigma}$ in three points $p_{1}, p_{2}, p_{3}$ (where $p_{1}, p_{3}$ are the end points of the arc). Then a bypass for $\Sigma$ (along $\alpha$ ), is a convex disk $D$ with Legendrian boundary such that

(1) $D \cap \Sigma=\alpha$,

(2) $t b(\partial D)=-1$,

(3) $\partial D=\alpha \cup \beta$,

(4) $\alpha \cap \beta=\left\{p_{1}, p_{3}\right\}$ are corners of $D$ and elliptic singularities of $D_{\xi}$.

The bypass attachment operation is the basic unit of isotopy of surfaces and will be crucial in our proofs. It is given in the following theorem.

Theorem 2.1 (Honda 2000, [17]). Let $\Sigma$ be a convex surface, $D$ a bypass for $\Sigma$ along a vertical $\alpha$ in $\Sigma$ (Figure 1), then there exists a neighborhood of $\Sigma \cup D \subset M$ diffeomorphic to $\Sigma \times[0,1]$, such that $\Sigma=\Sigma_{0}, \Sigma_{1}$ are convex, $\Sigma \times[0, \epsilon]$ is I-invariant and $\Gamma_{\Sigma}$ is related to $\Gamma_{\Sigma_{1}}$ as in Figure 2.

A surface $\Sigma$ locally separates the ambient manifold. If a bypass is contained in the (local) piece of $M \backslash \Sigma$ that has $\Sigma$ as its oriented boundary then we say the bypass will be attached to the front (or from the outside) of $\Sigma$ otherwise we say it is attached to the back (or from the inside) of $\Sigma$.

When a bypass is attached to a torus $T$ then either the dividing curves do not change and their number increases by two, or decreases by two, or the slope of the dividing curves changes. The slope of the dividing curves can change only when there are two dividing curves. If the bypass is attached to $T$ along a ruling curve then either the number of dividing curves decreases by two or the slope of the dividing curves changes. To understand the change in slope we need the following. Let $\mathbb{D}$ be the unit disk in $\mathbb{R}^{2}$. Recall the Farey tessellation of $\mathbb{D}$ is constructed as follows. Label the point $(1,0)$ on $\partial \mathbb{D}$ by $0=\frac{0}{1}$ and the point $(-1,0)$ with $\infty=\frac{1}{0}$. Now join them by a geodesic. If two points $\frac{p}{q}, \frac{p^{\prime}}{q^{\prime}}$ on $\partial \mathbb{D}$ with non-negative $y$-coordinate have been labeled, then label the point on $\partial \mathbb{D}$ half way between them (with non-negative $y$-coordinate) by $\frac{p+p^{\prime}}{q+q^{\prime}}$. Then connect this point to $\frac{p}{q}$ and to $\frac{p^{\prime}}{q^{\prime}}$ by a hyperbolic geodesic. Continue this until all positive fractions have been assigned to points on $\partial \mathbb{D}$ with non-negative $y$-coordinates. Now repeat this process for the points on $\partial \mathbb{D}$ with non-positive $y$-coordinate except start with $\infty=\frac{-1}{0}$. See Figure 3 . 

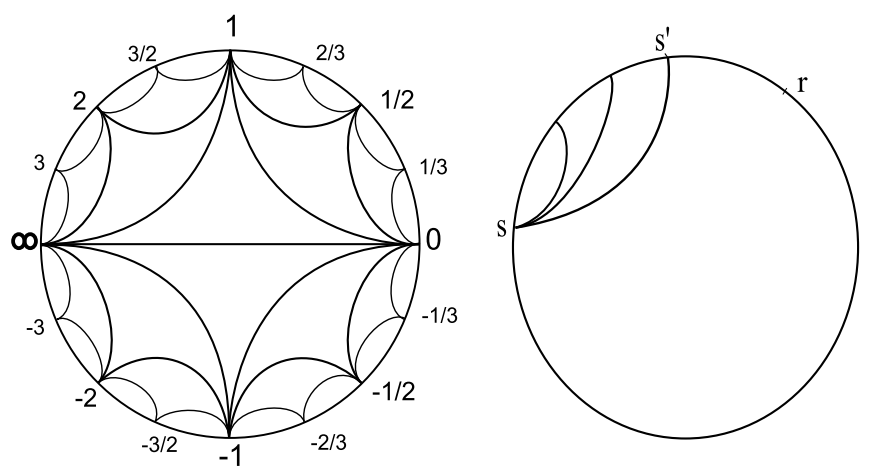

Figure 3. The Farey tessellation, on the left. Schematic of the change in the dividing slope from $s$ to $s^{\prime}$ after bypass attachment to the front of torus along a Legendrian ruling curve of slope $r$ on the Farey tessellation, on the right.

The key result we need to know about the Farey tessellation is given in the following theorem. See Figure 3.

Theorem 2.2 (Honda [17]). Let $T$ be a convex torus in standard form with $\left|\Gamma_{T}\right|=2$, dividing slope $s$ and ruling slope $r \neq s$. Let $D$ be a bypass for $T$ attached to the front of $T$ along a ruling curve. Let $T^{\prime}$ be the torus obtained from $T$ by attaching the bypass $D$. Then $\left|\Gamma_{T^{\prime}}\right|=2$ and the dividing slope $s^{\prime}$ of $\Gamma_{T^{\prime}}$ is determined as follows: let $[r, s]$ be the arc on $\partial \mathbb{D}$ running from $r$ counterclockwise to $s$, then $s^{\prime}$ is the point in $[r, s]$ closest to $r$ with an edge to $s$.

If the bypass is attached to the back of $T$ then the same algorithm works except one uses the interval $[s, r]$ on $\partial \mathbb{D}$.

2.1.3. The Imbalance Principle. As we see that bypasses are useful in changing dividing curves on a surface we mention a standard way to try to find them called the Imbalance Principle. Suppose that $\Sigma$ and $\Sigma^{\prime}$ are two disjoint convex surfaces and $A$ is a convex annulus whose interior is disjoint from $\Sigma$ and $\Sigma^{\prime}$ but its boundary is Legendrian with one component on each surface. If $\left|\Gamma_{\Sigma} \cdot \partial A\right|>\left|\Gamma_{\Sigma^{\prime}} \cdot \partial A\right|$ then there will be a dividing curve on $A$ that cuts a disk off of $A$ that has part of its boundary on $\Sigma$. It is now easy to use the Giroux Flexibility Theorem [15] to show that there is a bypass for $\Sigma$ on $A$.

2.1.4. Discretization of Isotopy. We will frequently need to analyze what happens to the contact geometry when we have a topological isotopy between two convex surfaces $\Sigma$ and $\Sigma^{\prime}$. This can be done by the technique of Isotopy Discretization [5] (see also [10] for its use in studying Legendrian knots). Given an isotopy between $\Sigma$ and $\Sigma^{\prime}$ one can find a sequence of convex surfaces $\Sigma_{1}=\Sigma, \Sigma_{2}, \ldots, \Sigma_{n}=\Sigma^{\prime}$ such that

(1) all the $\Sigma_{i}$ are convex and

(2) $\Sigma_{i}$ and $\Sigma_{i+1}$ are disjoint and $\Sigma_{i+1}$ is obtained from $\Sigma_{i}$ by a bypass attachment. Thus if one is trying to understand how the contact geometry of $M \backslash \Sigma$ and $M \backslash \Sigma^{\prime}$ relate, one just needs to analyze how the contact geometry of the pieces of $M \backslash \Sigma_{i}$ changes under bypass attachment. In particular, many arguments can be reduced from 
understanding a general isotopy to understanding an isotopy between two surfaces that cobound a product region.

There is also a relative version of Isotopy Discretization where $\Sigma$ and $\Sigma^{\prime}$ are convex surfaces with Legendrian boundary consisting of ruling curves on a convex torus. If $\partial \Sigma=\partial \Sigma^{\prime}$ and there is a topological isotopy of $\Sigma$ to $\Sigma^{\prime}$ relative to the boundary then we can find a discrete isotopy as described above. (Note that during the discrete isotopy the boundary of the surface is not fixed but is allowed to move among the ruling curves on the convex torus. One could slightly rephrase item (2) in the above definition of a discretized isotopy to keep the boundary fixed, but we find it more natural to allow the boundary to move even though the original isotopy is relative to the boundary.)

2.2. Standard neighborhood, transverse knots and stable simplicity. Given a Legendrian knot $L$, a standard neighborhood of $L$ is a solid torus $N$ that has convex boundary with two dividing curves of slope $1 / \operatorname{tb}(L)$ (and of course we will usually take $\partial N$ to be a convex torus in standard form). Conversely given any such solid torus it is a standard neighborhood of a unique Legendrian knot (cf. [19]).

One may understand stabilizations and destabilizations of a Legendrian knot $L$ in terms of the standard neighborhood. Specifically, inside the standard neighborhood $N$ of $L, L$ can be positively stabilized to $S_{+}(L)$, or negatively stabilized to $S_{-}(L)$. Let $N_{ \pm}$be a neighborhood of the stabilization of $L$ inside $N$. As above we can assume that $N_{ \pm}$has convex boundary in standard form. It will have dividing slope $\frac{1}{\operatorname{tb}(L)-1}$. Thus the region $N \backslash N_{ \pm}$is diffeomorphic to $T^{2} \times[0,1]$ and the contact structure on it is easily seen to be a basic slice; see [17]. There are exactly two basic slices with given dividing curves on their boundary and as there are two types of stabilization of $L$ we see that the basic slice $N \backslash N_{ \pm}$is determined by the type of stabilization done, and vice versa. Moreover, if $N$ is a standard neighborhood of $L$ then $L$ destabilizes if the solid torus $N$ can be thickened to a solid torus $N_{d}$ with convex boundary in standard form with dividing slope $\frac{1}{\operatorname{tb}(L)+1}$. Moreover, the sign of the destabilization will be determined by the basic slice $N_{d} \backslash N$. Finally, we notice that using Theorem 2.2 we can destabilize $L$ by finding a bypass for $N$ attached along a ruling curve whose slope is clockwise of $1 /(\operatorname{tb}(L)+1)$ (and anti-clockwise of 0 ).

Furthermore, by using this neighborhood one can talk about the positive/negative transverse push-off, $T_{ \pm}(L)$ of a Legendrian knot $L$. The only classical invariant of these transverse knots, the self-linking number, can be computed for transverse push-offs as (cf. [14])

$$
\operatorname{sl}\left(T_{ \pm}(L)\right)=\operatorname{tb}(L) \mp \mathrm{r}(L) .
$$

As in [10] two Legendrian knots $L$ and $L^{\prime}$ with the same tb and $\mathrm{r}$ are called stably isotopic if there is some $n$ and $n^{\prime}$ such that $S_{-}^{n}(L)$ and $S_{-}^{n^{\prime}}\left(L^{\prime}\right)$ are Legendrian isotopic. Note that $\operatorname{tb}(L)-\mathrm{r}(L)=\operatorname{tb}\left(S_{-}(L)\right)-\mathrm{r}\left(S_{-}(L)\right)$. A knot type $\mathcal{K}$ is called stably simple if Legendrian knots in this knot type are stably isotopic. The key result that we need concerning the transverse classification of a knot type is the following theorem of Epstein, Fuchs and Meyer from [14] (also [10] for general manifolds) which reduces the classification of transverse knots up to transverse isotopy to the classification Legendrian knots up to Legendrian isotopy and their negative stabilizations. 
Theorem 2.3 (Epstein-Fuchs-Meyer [14], Etnyre-Honda [10]). A knot type $\mathcal{K}$ is stably simple if and only if it is transversely simple.

2.3. Framings. One can talk about two coordinate systems for the boundary of a neighborhood of $\mathcal{K}_{(p, q)}$. In the first coordinate system, denoted by $\mathcal{C}$, the meridian has slope 0 and the well-defined longitude (coming from the intersection of a Seifert surface for $\mathcal{K}_{(p, q)}$ with $\left.\partial N\left(\mathcal{K}_{(p, q)}\right)\right)$ has slope $\infty$. In the second coordinate system, denoted $\mathcal{C}^{\prime}$, the meridian has slope 0 and slope $\infty$ comes from the surface $\partial N(\mathcal{K})$ on which $\mathcal{K}_{(p, q)}$ sits. As explained in [9] one can relate these two framings for $\partial N\left(\mathcal{K}_{(p, q)}\right)$ and deduce the following relation between the twisting of the contact planes along $L_{(p, q)}$ in $\mathcal{K}_{(p, q)}$ with respect to either framings.

$$
t\left(L_{(p, q)}, \mathcal{C}^{\prime}\right)+p q=t\left(L_{(p, q)}, \mathcal{C}\right)=\operatorname{tb}\left(L_{(p, q)}\right) .
$$

Given two embedded closed curves $\gamma$ and $\gamma^{\prime}$ on a torus $T$ we denote their minimal intersection by $\gamma \bullet \gamma^{\prime}$. If the slope of $\gamma$, respectively $\gamma^{\prime}$, is $s=\frac{r}{t}$, respectively $s^{\prime}=\frac{r^{\prime}}{t^{\prime}}$, then

$$
s \bullet s^{\prime}=\left|r t^{\prime}-t r^{\prime}\right| .
$$

The following two lemmas are from [9]. The first one is an easy consequence of equation (2.1) above.

Lemma 2.4. Let $L_{(p, q)}$ be a Legendrian knot in the knot type of $\mathcal{K}_{(p, q)}$.

(1) If $L_{(p, q)}$ is a Legendrian divide on $\partial N(\mathcal{K})\left(\right.$ in which case slope $\left.\left(\Gamma_{\partial N(\mathcal{K})}\right)=\frac{q}{p}\right)$, then

$$
\operatorname{tb}\left(L_{(p, q)}\right)=p q .
$$

(2) If $L_{(p, q)}$ is a Legendrian ruling curve on $\partial N(\mathcal{K})$ and slope $\left(\Gamma_{\partial N(\mathcal{K})}\right)=\frac{q^{\prime}}{p^{\prime}}$, then

$$
\operatorname{tb}\left(L_{(p, q)}\right)=p q-\left|\frac{p}{q} \bullet \frac{p^{\prime}}{q^{\prime}}\right| .
$$

Lemma 2.5. Let $D$ be a convex disk contained in $N(\mathcal{K})$ with Legendrian boundary on a contact isotopic copy of the convex surface $\partial N(\mathcal{K})$ and $\partial \Sigma(L)$ is the boundary of a convex Seifert surface of a Legendrian knot $L \in \mathcal{L}(\mathcal{K})$ which is contained in a contact isotopic copy of $\partial N(\mathcal{K})$. Then

$$
\mathrm{r}\left(L_{(p, q)}\right)=p \cdot \mathrm{r}(\partial D)+q \cdot \mathrm{r}(\partial \Sigma(L)) .
$$

\section{Legendrian simple cables}

In this section, we give the proofs of Theorems 1.1 and 1.3. But first we want to note that in the proofs we will, impicitly, use the following classical strategy, first proposed by Etnyre in [8] and efficiently used for almost all known results concerning the clasification of Legendrian knots.

(1) Find a formula that computes $\overline{\operatorname{tb}}\left(\mathcal{K}_{(p, q)}\right)$ and $\operatorname{r}(K)$ where $K \in \mathcal{L}\left(\mathcal{K}_{(p, q)}\right)$ with $\operatorname{tb}(K)=\overline{\operatorname{tb}}\left(\mathcal{K}_{(p, q)}\right)$.

(2) Classify Legendrian knots with maximal Thurston-Bennequin invariant. 
(3) Show that all Legendrian representatives of $\mathcal{K}_{(p, q)}$ of non-maximal ThurstonBennequin invariant admit destabilization or determine those that cannot be destabilized.

(4) Understand the relationship between the stabilizations of two non-destabilizable representatives of $\mathcal{K}_{(p, q)}$.

3.1. Sufficiently positive cables. We will work our way up to the proof of Theorem 1.1 through a series of lemmas.

Lemma 3.1. Under the hypothesis of Theorem 1.1, $\overline{\operatorname{tb}}\left(K_{(p, q)}\right)=p q-\left|\overline{\operatorname{tb}}(\mathcal{K}) \bullet \frac{p}{q}\right|$ and the set of rotation numbers realized by $L \in \mathcal{L}\left(K_{(p, q)}\right)$ with $\operatorname{tb}(L)=\overline{\operatorname{tb}}$ is

$$
\{q \cdot \mathrm{r}(K) \mid K \in \mathcal{L}(\mathcal{K}), \operatorname{tb}(K)=\overline{\operatorname{tb}}(K)\}
$$

Proof. During the proof we will use the $\mathcal{C}^{\prime}$ coordinate system. Note that $t w\left(L, \mathcal{C}^{\prime}\right)<0$ for all $L \in \mathcal{L}\left(K_{(p, q)}\right)$. If not, then there is an $L^{\prime} \in \mathcal{L}\left(K_{(p, q)}\right)$ with $t w\left(L^{\prime}\right)=0$, or equivalently, there exists a solid torus $S$ with $\partial S$ convex such that $L^{\prime}$ is a Legendrian divide on $\partial S$ which implies that slope of dividing set of $\partial S$ is $q / p$ when measured with respect to $\mathcal{C}$ but this contradicts the assumption that $\frac{p}{q}>\omega(\mathcal{K})$.

Let $S$ be a solid torus representing $\mathcal{K}$ with $\partial S$ convex, $L \subset \partial S$ and slope $\left(\Gamma_{\partial S}\right)=s$.

Recall in our Theorem 1.1 it is assumed that $\omega(\mathcal{K}) \in \mathbb{Z}$. Since $\overline{\operatorname{tb}}(\mathcal{K}) \leq \omega(\mathcal{K}) \leq$ $\overline{\operatorname{tb}}(\mathcal{K})+1$. We have either $\omega(\mathcal{K})=\overline{\operatorname{tb}}(\mathcal{K})$ or $\omega(\mathcal{K})=\overline{\operatorname{tb}}(\mathcal{K})+1$.

Case 1. $\omega(\mathcal{K})=\overline{\operatorname{tb}}(\mathcal{K})$ : We claim that the following inequality holds under the assumptions of Theorem 1.1:

$$
\left|\frac{1}{s} \bullet \frac{p}{q}\right| \geq\left|\omega(\mathcal{K}) \bullet \frac{p}{q}\right|
$$

and equality holds iff $\frac{1}{s}=\omega(\mathcal{K})$.

To see this note that, since $\omega(\mathcal{K}) \in \mathbb{Z}$ we know that on the Farey tesellesion there is an edge from 0 to $\frac{1}{\omega(\mathcal{K})}$. Moreover, by definition of the contact width we have $\frac{1}{s}<\omega(\mathcal{K})$. Now by using the oriented diffeomorphism of $\partial S$, we can normalize the slopes by sending 0 to 0 and $\frac{1}{\omega(\mathcal{K})}$ to $\infty$. This diffeomorphism will preserve order and hence force $q^{\prime} / p^{\prime}>0$ and $\frac{1}{s^{\prime}} \in(-\infty, 0)$ where $q^{\prime} / p^{\prime}$ and $\frac{1}{s^{\prime}}$ denote the images of $q / p$ and $\frac{1}{s}$ under the diffeomorphism, respectively.

Observe that $\frac{1}{s^{\prime}} \in(-\infty, 0)$ means

$$
\frac{1}{s^{\prime}}=m\left(\begin{array}{c}
0 \\
-1
\end{array}\right)+n\left(\begin{array}{l}
1 \\
0
\end{array}\right)=\left(\begin{array}{c}
n \\
-m
\end{array}\right)
$$

where $n, m>0$. Hence as slope $\frac{1}{s^{\prime}}=-\frac{m}{n}$. Now we easily get Inequality (3.1)

$$
\left|\frac{1}{s} \bullet \frac{p}{q}\right|=\left|\frac{1}{s^{\prime}} \bullet \frac{p^{\prime}}{q^{\prime}}\right|=\left|\frac{-m}{n} \bullet \frac{p^{\prime}}{q^{\prime}}\right|=\left|p^{\prime} n+q^{\prime} m\right|>p^{\prime}=\left|\frac{-1}{0} \bullet \frac{q^{\prime}}{p^{\prime}}\right|=\left|\omega(\mathcal{K}) \bullet \frac{p}{q}\right| .
$$


Therefore, $t\left(L, \mathcal{C}^{\prime}\right) \leq-\left|\omega(\mathcal{K}) \bullet \frac{p}{q}\right|$. Now any Legendrian ruling on $\partial S$, where $S$ is solid torus representing $\mathcal{K}$ of maximal thickness (i.e., slope $\left.\left(\Gamma_{\partial S}\right)=\frac{1}{\mathrm{tb}(\mathcal{K})}\right)$, realizes the equality. By equation (2.1) we see that

$$
\overline{\operatorname{tb}}\left(\mathcal{K}_{(p / q)}\right)=p q-|\omega(\mathcal{K}) \bullet p / q|=p q-|\overline{\operatorname{tb}}(\mathcal{K}) \bullet p / q| .
$$

Case 2. $\omega(\mathcal{K})=\overline{\operatorname{tb}}(\mathcal{K})+1$ : We first observe that $s=\frac{1}{\overline{\mathrm{tb}}(\mathcal{K})+1}$ is not possible as otherwise we would have $L \in \mathcal{L}(\mathcal{K})$ with $\operatorname{tb}(L)=\overline{\mathrm{tb}}(\mathcal{K})+1$. When $s \in\left[\frac{1}{\mathrm{tb}(\mathcal{K})}, \frac{1}{\overline{\mathrm{tb}}(\mathcal{K})+1}\right)$ the same proof as in Inequality (3.1) shows

$$
\left|\frac{1}{s} \bullet \frac{p}{q}\right| \geq\left|\overline{\operatorname{tb}}(\mathcal{K}) \bullet \frac{p}{q}\right| .
$$

and equality holds iff $\frac{1}{s}=\overline{\mathrm{tb}}(\mathcal{K})$.

Therefore $t\left(L, \mathcal{C}^{\prime}\right) \leq-\left|\overline{\operatorname{tb}}(\mathcal{K}) \bullet \frac{p}{q}\right|$ and any Legendrian ruling curve of slope $q / p$ on $\partial N$ with $s\left(\Gamma_{\partial N}\right)=\frac{1}{\operatorname{tb}(\mathcal{K})}$ will realize the equality in Inequality (3.2).

Next we compute the rotation numbers. Let $L \in \mathcal{L}\left(\mathcal{K}_{(p, q)}\right)$ with $\operatorname{tb}(L)=\overline{\operatorname{tb}}\left(\mathcal{K}_{(p, q)}\right)$. Such an $L$ is a Legendrian ruling curve on $\partial N$ where $N$ is a standard neighborhood of Legendrian knot $K \in \mathcal{L}(\mathcal{K})$ with $\operatorname{tb}(L)=\overline{\mathrm{tb}}$, i.e., slope $\left(\Gamma_{\partial N}\right)=\frac{1}{\mathrm{tb}(\mathcal{K})}$. Now, by formula (2.2) we have

$$
\mathrm{r}(L)=p \cdot \mathrm{r}(\partial D)+q \cdot \mathrm{r}(K)=q \cdot \mathrm{r}(K)
$$

as $\mathrm{r}(\partial D)=0$

Lemma 3.2. The $L \in \mathcal{L}\left(\mathcal{K}_{(p, q)}\right)$ with $\operatorname{tb}(L)=\overline{\mathrm{tb}}$ are classified by their rotation numbers.

Proof. If $L, L^{\prime} \in \mathcal{L}\left(\mathcal{K}_{(p, q)}\right)$ with $\operatorname{tb}(L)=\operatorname{tb}\left(L^{\prime}\right)=\overline{\mathrm{tb}}$, then there exist solid tori $S$ and $S^{\prime}$ which are standard neighborhood of $K, K^{\prime} \in \mathcal{L}(\mathcal{K})$ with $\operatorname{tb}(K)=\operatorname{tb}\left(K^{\prime}\right)=\overline{\operatorname{tb}(\mathcal{K})}$ , respectively. Since $t w(L, \partial S)<0$, we can make $\partial S$ convex (relative to $L$ ) and $L$ is Legendrian ruling curve on $\partial S$. Same things are true for $L^{\prime}$ and $S^{\prime}$.

If $\mathrm{r}(L)=\mathrm{r}\left(L^{\prime}\right)$, then by Lemma 3.1, $\mathrm{r}(K)=\mathrm{r}\left(K^{\prime}\right)$ and hence $K$ and $K^{\prime}$ are Legendrian isotopic by the Legendrian simplicity of the underlying knot type $\mathcal{K}$. Thus we may assume $K$ and $K^{\prime}$ are the same. Let $S$ and $S^{\prime}$ be the standard neighborhoods of the $K=K^{\prime}$ on which $L$ and $L^{\prime}$, respectively, sit. Since $K=K^{\prime} \subset S \cup S^{\prime}$, we can find a solid torus $S^{\prime \prime}$ sitting inside both $S$ and $S^{\prime}$ and with $\partial S^{\prime \prime}$ convex and slope $\left(\Gamma_{\partial S^{\prime \prime}}\right)=\frac{1}{\mathrm{tb}(\mathcal{K})}$. Since $\overline{S-S^{\prime \prime}}$ and $\overline{S^{\prime}-S^{\prime \prime}}$ are $I$-invariant neighborhoods, we can assume $L, L^{\prime}$ are (slope $q / p$ ) Legendrian rulings on $\partial S^{\prime \prime}$ which then are Legendrian isotopic through the other Legendrian rulings.

Remark 3.3. If the knot type $\mathcal{K}$ satisfies UTP property, then either there is single representative at maximal tb (hence has $\mathrm{r}=0$ ) or several representatives at maximal tb which are distinguished by their rotation numbers. Since in our case we are dealing with the knot types that do not necessarily satisfy UTP, there might be a knot type $\mathcal{K}$ that is Legendrian simple and has a Legendrian classification such that some $K^{\prime} \in \mathcal{L}(\mathcal{K})$ has $\operatorname{tb}\left(K^{\prime}\right)=n<\overline{\mathrm{tb}}$ but cannot be destabilized to $L$ with $\operatorname{tb}(L)=\overline{\mathrm{tb}}$. We note that Chongchitmate and $\mathrm{Ng}$ have conjectural examples in [4] of this phenomena. 
Lemma 3.4. For each non-destabilizable $K \in \mathcal{L}(\mathcal{K})$ with Thurston-Bennequin invariant $\operatorname{tb}(K)=n<\overline{\mathrm{tb}}$, there exists a unique, up to Legendrian isotopy, nondestabilizable $L, a(p, q)$-ruling curve on the standard neighborhood $N$ of $K$ with $\operatorname{tb}(L)=p q-\left|\frac{1}{n} \bullet \frac{p}{q}\right|$ and the set of rotation numbers associated with such $L$ is

$$
\{q \cdot \mathrm{r}(K) \mid K \in \mathcal{L}(\mathcal{K}), \operatorname{tb}(K)=n \text { and } K \text { is non-destabilizable }\} .
$$

Proof. Let $K \in \mathcal{L}(\mathcal{K})$ be such representative. Since $\operatorname{tb}(K)=n<\overline{\text { tb }}$ there exists an $L \in \mathcal{L}\left(K_{(p, q)}\right)$ which is a Legendrian ruling on $\partial N$ where $N$ is the standard neighborhood of $K \in \mathcal{L}(K)$, i.e., $s\left(\Gamma_{\partial N}\right)=\frac{1}{n}$ and $\# \Gamma_{\partial N}=2$. Now we want to show that $L$ does not admit a destabilization. Suppose $L$ admits a destabilization. This implies the existence of a convex torus $\Sigma$ which is (topologically) isotopic to $\partial N$ and contains $L$ and a bypass for $L$. Now isotope the annulus $A=\partial N-L$ to $A^{\prime}=\Sigma-L$ relative to the boundary $L$. By the Isotopy Discretization technique in [5] (cf. [18, Lemma 3.10]), we know such isotopy corresponds to a sequence of bypass attachments. Now we show that all potential bypass attachment are trivial, that is the dividing set of $A$ will not change and hence we cannot reach $A^{\prime}$. To end this, observe that a non-trivial bypass attachment from the outside corresponds to a thickening of $N$. Let $N^{\prime}$ denote the solid torus which corresponds to this thickening of $N$. Let $s\left(\Gamma_{\partial N^{\prime}}\right)=s$. Note that, $s\left(\Gamma_{\partial N^{\prime}}\right)=\frac{1}{n+1}$ is not possible since this will correspond to a destabilization of non-destabilizable $K \in \mathcal{L}(K)$. Hence if such thickening exist, then $\frac{1}{n+1}<s<\frac{1}{n}$. An important observation is that since bypass attachment happens in the complement of $L$, any bypass attachments to $A$ cannot increase the intersection number of the dividing set with $L$. On the other hand, as in Case 1 in Lemma 3.1, one can easily show for $s \in\left(\frac{1}{n+1}, \frac{1}{n}\right)$, the following inequality holds

$$
\left|\frac{q}{p} \bullet s\right|>\left|\frac{q}{p} \bullet \frac{1}{n}\right| .
$$

Thus, bypass attachment to $A$ from the outside must increase intersection number of the dividing set with $L$. Similarly one can show bypass attachment to $A$ from the inside would increase the intersection of the dividing set with $L$. Hence, we cannot reach $A^{\prime}$ and so $L$ does not destabilize.

Lemma 3.5. If $L \in \mathcal{L}\left(\mathcal{K}_{(p, q)}\right)$ with $\operatorname{tb}(L)<\overline{\operatorname{tb}}\left(K_{(p, q)}\right)$, then either $L$ admits a destabilization or $L$ is one of the non-destabilizable representative from Lemma 3.4.

Proof. Given such an $L$, there exists a solid torus $S$ with convex boundary, which contains $L$. Let $s\left(\Gamma_{\partial S}\right)=s$. If $L$ does not intersect the dividing set $\Gamma_{\partial S}$ efficiently, then we can destabilize $L$ with a bypass on $\partial S$. So we now assume that $L$ intersects $\Gamma_{\partial S}$ efficiently. Note that $s \neq \frac{1}{\omega(\mathcal{K})}$ as $\operatorname{tb}(L)<\overline{\operatorname{tb}}\left(\mathcal{K}_{(p, q)}\right)$. If $s=\frac{1}{n}$, then either $K \in \mathcal{L}(\mathcal{K})$ is non-destabilizable and we are in the situation of Lemma 3.4 or, as the underlying knot type $\mathcal{K}$ is Legendrian simple, $K \in \mathcal{L}(\mathcal{K})$ admits a destabilization which then gives a thickening of $S$, say $S^{\prime}$. Now, if the later one is the case, we can take a convex annulus $A=L \times[0,1]$ in $T^{2} \times[0,1]$, with $T_{0}=\partial S$ and $T_{1}=\partial S^{\prime}$, and use the Imbalance Principle to get a destabilization for $L$. Finally, suppose $s\left(\Gamma_{\partial S}\right)=$ $s \neq \frac{1}{n}$ and $S$ is non-thickenable. Shrink $S$ to a solid torus $S^{\prime \prime}$ with $\partial S^{\prime \prime}$ convex and 
$s\left(\Gamma_{\partial S^{\prime \prime}}\right)=\frac{1}{n^{\prime}}$ for some integer $n^{\prime}$. Again as in Case 1 in Lemma 3.1, we get that $\left|q^{\prime} / p^{\prime} \bullet s\right|=\left|q^{\prime} / p^{\prime} \bullet(-n / m)\right|=\left|p^{\prime} n+q^{\prime} m\right|>\left|p^{\prime} n-q^{\prime} n n^{\prime}\right|>\left|p^{\prime}-q^{\prime} n^{\prime}\right|=\left|q^{\prime} / p^{\prime} \bullet \frac{1}{n^{\prime}}\right|$, where $q^{\prime} / p^{\prime}$ denotes the image of $q / p$ under the diffeomorphism that takes 0 to 0 and $1 / n^{\prime}$ to $\infty$. Thus, we again get a destabilization for $L$.

Finally, we want to show for pairs (tb,r) obtained from stabilizations of multiple different non-destabilizable Legendrian knots (i.e., maximal tb representatives or Legendrian knots from Lemma 3.4), there is unique Legendrian with that tb and r. More precisely we prove

Lemma 3.6. If $L, L^{\prime} \in \mathcal{L}\left(\mathcal{K}_{(p, q)}\right)$ with $\operatorname{tb}(L)=\operatorname{tb}\left(L^{\prime}\right)=\overline{\operatorname{tb}}\left(\mathcal{K}_{(p, q)}\right)$ and $\mathrm{r}(L)=\mathrm{r}\left(L^{\prime}\right)+$ $2 q n$, then $S_{-}^{q n}(L)$ and $S_{+}^{q n}\left(L^{\prime}\right)$ are Legendrian isotopic. Also, if $\operatorname{tb}(L)=\overline{\operatorname{tb}}\left(\mathcal{K}_{(p, q)}\right)$ and $L^{\prime}$ is from Lemma 3.4 with $\mathrm{r}(L)=\mathrm{r}\left(L^{\prime}\right)+q(n-m)$, then $S_{-}^{q k}(L)$ and $S_{+}^{q l}\left(L^{\prime}\right)$, $k+l=n-m$, are Legendrian isotopic.

Proof. Observe that $L$ and $L^{\prime}$ sit on standard neighborhoods, say $N(K)$ and $N\left(K^{\prime}\right)$, of $K$ and $K^{\prime}$, respectively, where $K$ and $K^{\prime}$ of $\mathcal{L}(\mathcal{K})$ have maximal tb and $\mathrm{r}(K)=$ $\mathrm{r}\left(K^{\prime}\right)+2 n$, by the assumption and Lemma 3.1. As $\mathcal{K}$ is Legendrian simple, we have $S_{-}^{n}(K)=S_{+}^{n}\left(K^{\prime}\right)$. Let $N\left(S_{-}(K)\right)$ denote the standard neighborhood of $S_{-}(K)$ in $N(K)$. Let $L_{0}$ be a Legendrian ruling curve of slope $q / p$ on $\partial N\left(S_{-}(K)\right)$ and let $A$ be a convex annulus between $N(K)$ and $N\left(S_{-}(K)\right)$ with $L$ and $L_{0}$ being its boundary. A quick computation of tb shows that the dividing set on $A$ has to have $q$-boundary parallel $\operatorname{arcs}$ on $L_{0}$ side and no boundary parallel $\operatorname{arcs}$ on $L$ side (as otherwise we would be able to isotop $L$ along this bypass disks and end up with a representative with less twisting and contradict with the maximality of $L$ ). Now the boundary parallel arcs on $L_{0}$ side are all either positive or all negative, giving two kinds of destabilization of $L_{0}$. Therefore, we can easily conclude that $S_{-}^{q}(L)$ sits on a standard neighborhood of $S_{-}(K)$. In a similar way, it can be shown that $S_{+}^{q}\left(L^{\prime}\right)$ sits on the standard neighborhood of $S_{+}\left(K^{\prime}\right)$. We induct this argument to see that $S_{-}^{q n}(L)$ and $S_{+}^{q n}\left(L^{\prime}\right)$ sit on the standard neighborhoods of $S_{-}^{n}(K)=S_{+}^{n}\left(K^{\prime}\right)$. Now using the arguments as in the proof of Lemma 3.2, we conclude that $S_{-}^{q n}(L)$ and $S_{+}^{q n}\left(L^{\prime}\right)$ are Legendrian isotopic.

By using similar argument we can see that $L, L^{\prime} \in \mathcal{L}\left(\mathcal{K}_{(p, q)}\right)$ with $t b(L)=\overline{\mathrm{tb}}$ and $L^{\prime}$ is from Lemma 3.4 and $\mathrm{r}(L)=\mathrm{r}\left(L^{\prime}\right)+q(n-m)$ stabilize to same Legendrian knot.

Proof of Theorem 1.1. Lemmas 3.2, 3.4 and 3.5 give a complete list of non-destabilizable Legendrian knots in $\mathcal{K}_{(p, q)}$ and they are all determined by tb and r, by Lemma 3.6.

3.2. Sufficiently negative cables. Now we give the proof of Theorem 1.3. The proof is established through the following sequence of lemmas.

Lemma 3.7. Under the hypothesis of Theorem 1.3, $\overline{\operatorname{tb}}\left(\mathcal{K}_{(p, q)}\right)=p q=\omega\left(\mathcal{K}_{(p, q)}\right)$ and the set of rotation numbers realized by $L_{(p, q)} \in \mathcal{L}\left(\mathcal{K}_{(p, q)}\right)$ with $\operatorname{tb}(L)=\overline{\operatorname{tb}}\left(\mathcal{K}_{(p, q)}\right)$ is

$$
\{ \pm(p+q(n+r(L)): L \in \mathcal{L}(\mathcal{K}), \operatorname{tb}(L)=-n\}
$$

where $n$ is the integer that satisfies

$$
-n-1<\frac{p}{q}<-n .
$$


Proof. We will use the $\mathcal{C}^{\prime}$ coordinate system. Observe that since $\frac{p}{q}<\ell \omega(\mathcal{K})$, there is a convex torus $T=\partial N$ of dividing slope $q / p$ inside the solid torus $S$ and parallel to $\partial S$, where $S$ represents $\mathcal{K}$ with $\partial S$ convex and has dividing slope $1 / \ell \omega(\mathcal{K})$. Now a Legendrian divide on $T$ is a representative $L_{(p, q)} \in \mathcal{L}\left(\mathcal{K}_{(p, q)}\right)$ with twisting number zero. Thus $\bar{t}\left(\mathcal{K}_{(p, q)}, \mathcal{C}^{\prime}\right) \geq 0$.

For the equality it is enough to show that $\omega\left(\mathcal{K}_{(p, q)}, \mathcal{C}^{\prime}\right)=0$ since $\bar{t}\left(\mathcal{K}_{(p, q)}, \mathcal{C}^{\prime}\right) \leq$ $\omega\left(\mathcal{K}_{(p, q)}, C^{\prime}\right)$. The proof below is essentially Claim 4.2 in [9]. The key point is showing that the knot type $\mathcal{K}_{(p, q)}$ satisfies the first condition of the UTP.

Let $N_{(p, q)}$ be a solid torus representing $\mathcal{K}_{(p, q)}$ and has convex boundary with $s\left(\Gamma_{\partial\left(N_{(p, q)}\right)}=s\right.$. We want to show $s=0$. Suppose $s>0$. After thinning the solid tori $N_{(p, q)}$, if necessary, we may take $s$ to be a large positive integer and $\# \Gamma_{\partial\left(N_{(p, q)}\right.}=2$. We use Giroux's Flexibility Theorem [15], to arrange characteristic foliation on $\partial N_{(p, q)}$ to be in standard form and Legendrian ruling to have slope $\infty$. Consider a convex annulus $A$ with Legendrian boundary of slope $\infty$ on $\partial N_{(p, q)}$ such that a thickening $R=N_{(p, q)} \cup(A \times[-\epsilon, \epsilon]) \cong T^{2} \times[1,2]$ has $\partial R=T_{1} \cup T_{2}$ parallel to $N(\mathcal{K})$, where $N(\mathcal{K})$ is a solid torus representing $\mathcal{K}$ with convex boundary of dividing slope $q / p$. Moreover, with this setting, $T_{2} \supset \partial N$ and $T_{1} \subset N(\mathcal{K})$. Note that $\Gamma_{A}$ must consist of parallel non-separating arcs, otherwise we can attach the bypass corresponding to boundary parallel arcs onto $\partial\left(N_{(p, q)}\right)$ to increase $s$ to $\infty$ by Theorem 2.2 . This will result in excessive twisting inside $N\left(\mathcal{K}_{(p, q)}\right)$ and hence would result in contact structure to be overtwisted. Moreover, we can take an identification of $\partial N(\mathcal{K}) \cong \mathbb{R}^{2} / \mathbb{Z}^{2}$ so that slope $\left(\Gamma_{T_{1}}\right)=-s$ and slope $\left(\Gamma_{T_{2}}\right)=1$. To see this, we note that $T_{1}$ and $T_{2}$ are each obtained by gluing one half of $\partial N\left(\mathcal{K}_{(p, q)}\right)$ to the annulus $A$ and now since $s$ is a positive integer, it is clear that $\Gamma_{T_{1}}$ is obtained from $\Gamma_{T_{2}}$ by performing $s+1$ right-handed Dehn twists.

Let $N^{\prime}$ be a solid torus of maximal thickness containing $R$. By [17, Proposition 5.1], such a neighborhood has only universally tight contact structures. Note that any tight contact structure on $R$ can be layered into two basic slices at the torus $T_{1.5}$ parallel to $T_{i}, i=1,2$, with $\operatorname{slope}\left(\Gamma_{T_{1.5}}\right)=\infty$ which is $q / p$ when measured with respect to $\mathcal{C}$ coordinate system. A quick computation of the Poincaré duals of the relative Euler classes for each of this basic slices shows that there are four possible tight contact structures on $R$ (two for each basic slice), which are given by $\pm(1,0) \pm(1,1-s)$ and the universally tight ones has no mixing of sign (i.e., either $+(1,0)+(1,1-s)$ or $-(1,0)-(1,1-s))$. We want to determine if the tight contact structure $\xi$ we start with on $R$, has a mixing of sign or not. To end this, we compute the Euler class. Let $\gamma$ be a Legendrian ruling curve of slope $\infty$ on $A$ and let $A^{\prime}=\gamma \times[-\epsilon, \epsilon]$. We easily see that the dividing set on $A^{\prime}$ is made of $2 s$ parallel curves (as $A^{\prime}$ is $(-\epsilon, \epsilon)$ invariant), we use this to get that $<e(\xi), A^{\prime}>=\chi\left(A_{+}^{\prime}\right)-\chi\left(A_{-}^{\prime}\right)=0$, this gives then $P D e(\xi)= \pm(0,1-s)$. So, there is a mixing of sign. But this cannot happen inside $N^{\prime}$. Thus, $s=0$ and we get $\omega\left(\mathcal{K}_{(p, q)}, \mathcal{C}^{\prime}\right)=0$, passing $\mathcal{C}$ coordinate system we have $\overline{\operatorname{tb}}\left(\mathcal{K}_{(p, q)}\right)=p q$.

Now we want to compute rotation numbers of $L_{(p, q)}$ in $\mathcal{L}\left(\mathcal{K}_{(p, q)}\right)$ realizing the maximal Thurston-Bennequin number. Let $T_{1.5}^{2}=\partial N$ which contains $L_{(p, q)}$ with $\operatorname{tb}\left(L_{(p, q)}\right)=p q$. Since $\frac{p}{q}<\ell \omega(\mathcal{K})$, we can take a thickening of tori $T_{1.5}^{2}, T^{2} \times[1,2]$ such that boundary tori have slope $\operatorname{slope}\left(\Gamma_{T_{1}^{2}}\right)=-\frac{1}{n-1}$ and $\operatorname{slope}\left(\Gamma_{T_{1}^{2}}\right)=-\frac{1}{n}$ where 
$n$ is the integer that satisfies $-n-1<\frac{p}{q}<-n$ (note that $n$ may equal to $\ell \omega(\mathcal{K})$ ). But now the solid tori of boundary slopes $-\frac{1}{n-1}$ and $-\frac{1}{n}$ are the standard neighborhoods of $L$ and $S_{ \pm}(L)$, respectively. We can now make the relative Euler class computation as above and then use Lemma 2.5 to get desired formula for the rotation number computation.

Lemma 3.8. Legendrian knots with maximal tb in $\mathcal{L}\left(\mathcal{K}_{(p, q)}\right)$ are determined by their rotation numbers.

Proof. Let $L$ and $L^{\prime}$ be two Legendrian knots in $\mathcal{L}(\mathcal{K})$ with maximal tb and $\mathrm{r}(L)=$ $\mathrm{r}\left(L^{\prime}\right)$, then we have associated solid tori $N$ and $N^{\prime}$ with convex boundary on which $L$ and $L^{\prime}$ sit as Legendrian divides. The classification of tight contact structures on the solid torus in $[16,17]$ says that the contactomorphism type of a tight contact structure on a solid torus with convex boundary is determined by the number of the positive bypasses on the meridional disk. Hence, determined by the rotation number of $L$ and $L^{\prime}$, respectively, which are the same by the assumption. Thus, we get a contactomorphism $f: N \rightarrow N^{\prime}$. We may extend $f$ to a contactomorphism of $S^{3}$ that takes $\partial N$ to $\partial N^{\prime}$. Furthermore, by using Eliashberg's result in [6], there is indeed a contact isotopy of $S^{3}$ that takes $\partial N$ to $\partial N^{\prime}$. So, we will now think that $L$ and $L^{\prime}$ are Legendrian divides on the convex boundary of the same solid torus, say $N$. We are left to form a Legendrian isotopy between $L$ and $L^{\prime}$. To end this, we recall from Lemma 3.7 that $\partial N$ is siting inside a thickened torus $T^{2} \times[1,2]$ such that the boundary tori have slope $\left(\Gamma_{T_{1}^{2}}\right)=-\frac{1}{n-1}$ and $\operatorname{slope}\left(\Gamma_{T_{2}^{2}}\right)=-\frac{1}{n}$. Now as the consequence of the classification of tight contact structure on thickened tori (see [17, Corollary 4.8]), we know that there is also a pre-Lagrangian torus, (still) denote by $\partial N$, which has linear foliation and the same boundary slope as convex torus does. Moreover (after contact isotopy) our convex torus intersect with the pre-Lagrangian torus transversely and exactly in Legendrian divides. Thus, we can take $L$ and $L^{\prime}$ to be two leaves on this linearly foliated (pre-Lagrangian) torus. Now, $L$ and $L^{\prime}$ are Legendrian isotopic through this linear characteristic foliation.

Lemma 3.9. If $L^{\prime} \in \mathcal{K}_{(p, q)}$ with $\operatorname{tb}\left(L^{\prime}\right)<\overline{\mathrm{tb}}$, then $L^{\prime}$ admits a destabilization.

Proof. We can put $L^{\prime}$ on a solid torus $S$ with $\partial S$ convex and $\operatorname{slope}\left(\Gamma_{\partial S}\right)=s$. By the Lemma 3.8 and the assumption that $\frac{p}{q}<\ell \omega(\mathcal{K})$ we can deduce that $L^{\prime}$ is a Legendrian ruling on $S$ (clearly we can assume $L^{\prime}$ intersects $\Gamma_{\partial S}$ efficiently otherwise destabilization is immediate) and $\frac{1}{s} \neq \ell \omega(\mathcal{K})$. If $s<\frac{1}{\ell \omega(\mathcal{K})}$, then, as in equation (3.1), we easily see that $|q / p \bullet s|>|q / p \bullet 1 / \ell \omega|$. Hence, by using the Imbalance Principle, we get a destabilization of $L^{\prime}$. If $s>\frac{1}{\ell \omega(\mathcal{K})}$, then we can thicken $S$ to a solid tori $S^{\prime}$ with $\partial S^{\prime}$ convex and $\operatorname{slope}\left(\Gamma_{\partial S^{\prime}}\right)=\frac{1}{\ell \omega(\mathcal{K})}$. Hence, taking a convex annulus $A$ with one boundary component on $L^{\prime}$ in $\partial S \times[0,1]=\overline{S^{\prime}-S}$ and applying the Imbalance Principle again we find a bypass for $L^{\prime}$ which gives a destabilization for $L^{\prime}$.

Lemma 3.10. If $L_{(p, q)}^{+}, L_{(p, q)}^{-} \in \mathcal{L}\left(\mathcal{K}_{(p, q)}\right)$ with $\operatorname{tb}\left(L_{(p, q)}^{+}\right)=\operatorname{tb}\left(L_{(p, q)}^{-}\right)$and $\mathrm{r}\left(L_{(p, q)}^{+}\right)=$ $\mathrm{r}\left(L_{(p, q)}^{-}\right)+2 p+2 q n\left(\right.$ or $\left.\mathrm{r}\left(L_{(p, q)}^{+}\right)=\mathrm{r}\left(L_{(p, q)}^{-}\right)+2 k q-2 p-2 q n\right)$, then $S_{+}^{p+q n}\left(L_{(p, q)}^{-}\right)=$ $S_{-}^{p+q n}\left(L_{(p, q)}^{+}\right)\left(\right.$or $\left.S_{+}^{k q-p-q n}\left(L_{(p, q)}^{-}\right)=S_{-}^{k q-p-q n}\left(L_{(p, q)}^{+}\right)\right)$. 
Proof. There are two cases of concern based on rotation number computation in Lemma 3.7.

Case 1: $L \in \mathcal{L}(\mathcal{K})$ in Lemma 3.7 has $\mathrm{r}(L)=0$. In this case, $L_{(p, q)}^{ \pm}$are the only maximal tb representatives of $\mathcal{L}\left(\mathcal{K}_{(p, q)}\right)$ with $\mathrm{r}\left(L_{(p, q)}^{+}\right)=-p-q n$ and $\mathrm{r}\left(L_{(p, q)}^{-}\right)=$ $p+q n$. Clearly by doing $-p-q n$ positive (respectively negative) stabilization on $L_{(p, q)}^{-}$ (respectively on $\left.L_{(p, q)}^{+}\right)$we end up at Legendrian knots with the same (tb, r) pair. We also have $L_{(p, q)}^{\prime} \in \mathcal{L}\left(\mathcal{K}_{(p, q)}\right)$ with $\operatorname{tb}\left(L_{(p, q)}^{\prime}\right)=\overline{\operatorname{tb}}\left(\mathcal{K}_{(p, q)}\right)+p+q n$ and $\operatorname{r}\left(L_{(p, q)}^{\prime}\right)=$ $q \mathrm{r}(L)=0$. We know by Lemma 3.9, such an $L_{(p, q)}^{\prime}$ admits a destabilization. We want to show that these are all Legendrain isotopic, i.e., $S_{+}^{-p-q n}\left(L_{(p, q)}^{+}\right)=L_{(p, q)}^{\prime}=$ $S_{-}^{-p-q n}\left(L_{(p, q)}^{-}\right)$. Recall that $L_{(p, q)}^{ \pm}$are the Legendrian divide on a convex torus $T_{1.5}$ with boundary slope $\frac{q}{p}$ inside $T^{2} \times[1,2]=N(L)-N\left(S_{ \pm}(L)\right)$ (see the remark at the end of the statement of $\left[9\right.$, Lemma 3.8]). Hence, $L_{(p, q)}^{\prime}$ is a Legendrian ruling curve of slope $\frac{q}{p}$ on the standard neighborhood $N(L)$ of $L \in \mathcal{L}(\mathcal{K})$ with $\operatorname{tb}(L)=-n$. Note that, $S_{+}^{-p-q n}\left(L_{(p, q)}^{+}\right)$and $S_{-}^{-p-q n}\left(L_{(p, q)}^{-}\right)$are also Legendrian ruling curve on $N(L)$. Hence, $L_{(p, q)}^{\prime}$ is Legendrian isotopic to $S_{+}^{-p-q n}\left(L_{(p, q)}^{+}\right)$and $S_{-}^{-p-q n}\left(L_{(p, q)}^{-}\right)$through the other ruling curves. Indeed, by taking a convex annulus $A=L_{(p, q)} \times[1.5,2]$ between $T_{1.5}$ and $N(L)$ with $\partial A$ is Legendrian curves of slope $\frac{q}{p}$ on $T_{1.5}$ and $N(L)$, we easily see $L_{(p, q)}^{\prime}$ destabilizes in two ways.

Case 2: $L \in \mathcal{L}(\mathcal{K})$ in Lemma 3.7 has $\mathrm{r}(L) \neq 0$. In this case, $L_{(p, q)}^{ \pm} \in \mathcal{L}\left(\mathcal{K}_{(p, q)}\right)$ corresponds to $L^{ \pm} \in \mathcal{L}(\mathcal{K})$ where $\operatorname{tb}\left(L^{+}\right)=\operatorname{tb}\left(L^{-}\right)=-n$ and $\mathrm{r}\left(L^{+}\right) \neq \mathrm{r}\left(L^{-}\right)$. Without loss generality we can assume that $\mathrm{r}\left(L^{-}\right)<\mathrm{r}\left(L^{+}\right)$and there is no $L^{0}$ with $\mathrm{r}\left(L^{-}\right)<\mathrm{r}\left(L^{0}\right)<\mathrm{r}\left(L^{+}\right)$, then $\mathrm{r}\left(L^{+}\right)-\mathrm{r}\left(L^{-}\right)=2 k, k \in \mathbb{Z}_{>0}$. Thus, $\mathrm{r}\left(L_{(p, q)}^{-}\right)=$ $q \mathrm{r}\left(L^{-}\right)+p+q n$ and $\mathrm{r}\left(L_{(p, q)}^{+}\right)=q \mathrm{r}\left(L^{+}\right)-p-q n=q \mathrm{r}\left(L^{-}\right)-(2 k q+p+q n)$. This extra depth $k q$ comes from the underlying knot type puts us precisely in the situation of Lemma 3.6. Namely, the $L_{(p, q)}^{\prime}$ with $\operatorname{tb}\left(L_{(p, q)}^{\prime}\right)=p q-(k q+p+q n)$ and $\mathrm{r}\left(L_{(p, q)}^{\prime}\right)=q \mathrm{r}\left(L^{+}\right)+k q=q \mathrm{r}\left(L^{-}\right)-k q$ is the Legendrian ruling curve of slope $\frac{q}{p}$ on the standard neighborhood $S_{+}^{k}\left(L^{+}\right)=S_{-}^{k}\left(L^{-}\right)$(as $\mathcal{K}$ is Legendrian simple). Therefore, a Legendrian isotopy through the other ruling curves gives that $L_{(p, q)}^{\prime}=S_{+}^{k q-p-q n}\left(L_{(p, q)}^{-}\right)=S_{-}^{k q-p-q n}\left(L_{(p, q)}^{+}\right)$.

Proof of Theorem 1.3. Lemmas 3.7 and 3.8 give a complete list of non-destabilizable Legendrian knots in $\mathcal{K}_{(p, q)}$ and show that they are all determined by their tb and $\mathrm{r}$. By Lemma 3.9, every $L_{(p, q)}^{\prime}$ in $\mathcal{L}\left(\mathcal{K}_{(p, q)}\right)$ with non-maximal tb invariant can be written as $S_{-}^{k} S_{+}^{l}\left(L_{(p, q)}\right)$ for some $L_{(p, q)}^{ \pm} \in \mathcal{L}\left(\mathcal{K}_{(p, q)}\right)$ with maximal tb. Finally, Lemma 3.10 shows any two $L_{(p, q)}^{ \pm}$with maximal tb and $\mathrm{r}\left(L_{(p, q)}^{-}\right)<\mathrm{r}\left(L_{(p, q)}^{+}\right)$(and no $L_{(p, q)}^{0}$ with $\left.\mathrm{r}\left(L_{(p, q)}^{-}\right)<\mathrm{r}\left(L_{(p, q)}^{0}\right)<\mathrm{r}\left(L_{(p, q)}^{+}\right)\right)$, stabilize to same $L_{(p, q)}^{\prime}$ in $\mathcal{L}\left(\mathcal{K}_{(p, q)}\right)$.

\section{Concluding remark}

We want to note that Corollary 1.4 does not immediately follow from Theorems 1.1 and 1.3. More precisely, we can conclude the Legendrian simplicity of positive torus knots from Theorem 1.1 but the Legendrian simplicity of negative torus knots does not follow from Theorem 1.3; however, it does follow from the proof of Theorem 1.3 as 
we now indicate. When $\ell \omega(\mathcal{K})=-\infty$ there are no non-thickenable tori so any torus of slope less than $\omega(\mathcal{K})$ will thicken. In particular, given any slope $p / q<\omega(K)$ there is some solid torus in the knot type $\mathcal{K}$ with dividing curves on its boundary having slope $p / q$ and thus we can conclude $\bar{t}\left(\mathcal{K}_{(p, q)}, C^{\prime}\right) \geq 0$. In the case of the unknot, one can now conclude that $\omega\left(K_{(p, q)}, C^{\prime}\right)=0$ as in the proof of Theorem 1.3 and the rest of the proof also carries over to this case too.

We finish with the following question which we believe has a good chance to be answered positively.

Question 4.1. Let $\mathcal{K}$ be a knot type in $S^{3}$ with $\ell \omega(\mathcal{K})=-\infty$. Is it true that, $\mathcal{K}$ has to be the unknot?

\section{Acknowledgments}

The author is grateful to John Etnyre for his continuous support and guidance. The author would also like to thank Douglas LaFountain, Vera Vertesi and Lenny Ng for helpful discussions. The author was partially supported during the course of this work by TÜBİTAK, the Scientific and Technological Research Council of Turkey and NSF Grant DMS-0804820.

\section{References}

[1] K.L. Baker, J.B. Etnyre, and J. Van Horn-Morris, Cabling, rational open book decompositions and contact structures, J. Differ. Geom., 90 (2012), 1-80.

[2] D. Bennequin, Entralacements et équations de Pfaff, Astérisque, 107-108 (1983), 87-161.

[3] J. Birman and W. Menasco, Stabilization in the braid groups II: transverse simplicity of transverse knots, Geom. \& Topol., 10 (2006), 1425-1452.

[4] W. Chongchitmate and L. Ng, An atlas of Legendrian knots, arXiv: 1010.3997, 2010.

[5] V. Colin, Chirurgies d'indice un et isotopies de sphères dans les variétés de contact tendues, C. R. Acad. Sci. Paris Sér. I Math., 324(6) (1997), 659-663.

[6] Y. Eliashberg, Contact 3-manifolds twenty years since J. Martinet's work., Geometry of low dimensional manifolds, volume II (Donaldson and Thomas Ed.), Cambridge, 1990.

[7] Y. Eliashberg and M. Fraser, Classification of topologically trivial Legendrian knots, in 'Geometry, topology, and dynamics' (Montreal, PQ, 1995), pages 1751, CRM Proc. Lecture Notes, 15, 1998.

[8] J.B. Etnyre, Transversal torus knots, Geom. \& Topol., 3 (1999), 253-268.

[9] J.B. Etnyre and K. Honda, Cabling and Transverse simplicity, Ann. Math., 162(2) (2005), $1305-1333$.

[10] J.B. Etnyre and K. Honda, Knots and contact geometry I: torus knots and the figure eight knot. J. Symplectic Geom., 1(2001), 63-120

[11] John B. Etnyre and Ko Honda, On connected sums and Legendrian knots, Adv. Math., 179(1) (2003), 59-74.

[12] J.B. Etnyre, D. LaFountain, and B. Tosun, Legendrian and transverse cables of positive torus knots, Geom. Topol., 16 (2012) 1639-1689

[13] J.B. Etnyre, Lenny $\mathrm{Ng}$, and Vera Vértesi, Legendrian and transverse twist knots. arXiv:1002.2400, 2010.

[14] J. Epstein, D. Fuchs, and M. Meyer, Chekanov-Eliashberg invariants and transverse approximations of Legendrian knots, Pacific J. Math., 201(1) (2001), 89-106.

[15] E. Giroux, Convexité en topologie de contact, Comment. Math. Helv., 66 (1991), 637-677.

[16] E. Giroux, Structures de contact en dimension trois et bifurcations des feuilletages de surfaces, Invent. Math., 141 (2000), 615-689.

[17] K. Honda, On the classification of tight contact structures I, Geom. Topol., 4 (2000), 309-368. Factoring nonrotative $T^{2} \times I$ layers, Erratum to "On the classification of tight contact structures I", Geometry \& Topology, 5 (2001), 925-938. 
[18] K. Honda, Gluing tight contact structures, Duke Math. J., 115 (2002), 435-478.

[19] Y. Kanda, The classification of tight contact structures on the 3-torus, Comm. Anal. Geom., 5 (1997), 413-438.

[20] D.J. LaFountain, Studying uniform thickness I: Legendrian simple iterated torus knots, Alg. Geom. Top., 10(2) (2010), 891-916 (electronic).

Department of Mathematics, University of Virginia, Charlottesville, VA 22904

E-mail address: bt5t@virginia.edu

$U R L:$ http:///people.virginia.edu/bt5t 
